\title{
The effects of bisphosphonate and radiation therapy in bone- metastatic lung adenocarcinoma: the impact of KRAS mutation
}

\author{
Peter Radeczky ${ }^{1,2}$, Zsolt Megyesfalvi ${ }^{1,2,3}$, Viktoria Laszlo ${ }^{2,3}$, Janos Fillinger $^{1,2}$, Judit Moldvay ${ }^{2,4}$ Erzsebet Raso $^{5}$, \\ Erzsebet Schlegl' ${ }^{2}$, Tamas Barbai ${ }^{5}$, Jozsef Timar ${ }^{5,6}$, Ferenc Renyi-Vamos ${ }^{1,2}$, Balazs Dome ${ }^{1,2,3 *}$, Balazs Hegedus ${ }^{7 *}$ \\ ${ }^{1}$ Department of Thoracic Surgery, National Institute of Oncology-Semmelweis University, Budapest, Hungary; ${ }^{2}$ National Koranyi Institute of \\ Pulmonology, Budapest, Hungary; ${ }^{3}$ Department of Thoracic Surgery, Comprehensive Cancer Center, Medical University of Vienna, Vienna, Austria; \\ ${ }^{4}$ MTA-SE NAP, Brain Metastasis Research Group, Hungarian Academy of Sciences, Budapest, Hungary; ${ }^{5}$ 2nd Department of Pathology, Semmelweis \\ University, Budapest, Hungary; ${ }^{6}$ Tumor Progression Research Group, Hungarian Academy of Sciences-Semmelweis University, Budapest, Hungary; \\ ${ }^{7}$ Department of Thoracic Surgery, Ruhrlandklinik, University Clinic Essen, Essen, Germany \\ Contributions: (I) Conception and design: P Radeczky, Z Megyesfalvi, J Timar, B Dome, B Hegedus; (II) Administrative support: P Radeczky, B \\ Hegedus; (III) Provision of study materials or patients: P Radeczky, J Moldvay, V Laszlo, J Fillinger, F Renyi-Vamos; (IV) Collection and assembly of \\ data: E Raso, T Barbai, E Schlegl, P Radeczky, Z Megyesfalvi, J Timar; (V) Data analysis and interpretation: P Radeczky, Z Megyesfalvi, B Hegedus; \\ (VI) Manuscript writing: All authors; (VII) Final approval of manuscript: All authors. \\ *These authors contributed equally to this work as senior authors. \\ Correspondence to: Balazs Hegedus, PhD. Department of Thoracic Surgery, Ruhrlandklinik, University Duisburg-Essen, 45239, Essen, Germany. \\ Email: balazs.hegedues@rlk.uk-essen.de; Balazs Dome, MD, PhD. Department of Thoracic Surgery, Comprehensive Cancer Center, Medical \\ University of Vienna, Vienna, Austria. Email: balazs.dome@meduniwien.ac.at.
}

Background: KRAS mutation is the most common genetic alteration in lung adenocarcinoma (LADC) in Western countries and is associated with worse outcome in bone-metastatic cases. Yet, to date, no effective treatment guidelines were developed for these patients. Accordingly, our aim was to investigate the impact of KRAS mutation on bisphosphonate (BTx) and radiation therapy (RTx) in bone-metastatic LADC patients.

Methods: Clinicopathological variables of 134 consecutive LADC patients with bone metastases at diagnosis and known KRAS status were retrospectively analyzed. The effects of BTx, RTx and KRAS mutation on overall survival (OS) were investigated.

Results: Of the total cohort, 93 patients were identified as KRAS wild-type (WT) (69.4\%) and 41 (30.6\%) as KRAS mutant patients. The presence of KRAS mutation was associated with significantly reduced median OS (5.1 vs. 10.2 months in KRAS WT patients; $\mathrm{P}=0.008$ ). Irrespective of KRAS mutational status both $\mathrm{BTx}$ $(\mathrm{P}=0.007)$ and RTx $(\mathrm{P}=0.021)$ conferred a significant benefit for OS. Notably, however, when analyzing the patients with KRAS-mutant and KRAS WT tumors separately, the benefit from BTx and RTx on OS remained statistically significant only in KRAS WT patients ( $\mathrm{P}=0.032$ and $\mathrm{P}=0.031$, respectively).

Conclusions: KRAS mutation is a strong negative prognostic factor in bone-metastatic LADC patients. Both BTx and RTx can increase the OS with a pronounced benefit for patients with KRAS WT tumors. Altogether, KRAS mutational status should be considered during therapeutic decision making in bonemetastatic LADC patients.

Keywords: Bone metastases; lung adenocarcinoma (LADC); KRAS mutation; bisphosphonate therapy; radiation therapy

Submitted Jun 17, 2020. Accepted for publication Nov 23, 2020.

doi: $10.21037 /$ tlcr-20-754

View this article at: http://dx.doi.org/10.21037/tlcr-20-754 


\section{Introduction}

Lung cancer remains the most frequently diagnosed cancer worldwide (11.6\% of the total cases) and the leading cause of cancer-related deaths $(18.4 \%$ of the total cancer deaths) (1). Histologically, the most common type of lung cancer is adenocarcinoma [lung adenocarcinoma (LADC)], comprising around up to $40 \%$ to $50 \%$ of all lung cancer cases $(2,3)$. Unfortunately, the majority of patients already have advanced-stage disease at diagnosis with different types of distant organ metastases (4).

Bone is a common site of metastatic cancer spread in LADC since about $25-40 \%$ of all advanced-stage LADC patients develop skeletal metastases during the course of their disease (5-7). These metastases are associated with short overall survival (OS) (usually less than 1 year from diagnosis), loss of functional independence and reduction in quality of life $(5,7)$. Accordingly, bone metastases are often complicated by skeletal-related events (SREs), including pathological fractures, spinal cord compression, and hypercalcemia (5). It is not surprising therefore that there is an urgent necessity for the development of improved therapeutic strategies in order to mitigate the deaths and disabilities caused by bone metastases. To date, bisphosphonates are one of the most commonly used therapeutic agents to prevent and reduce the incidence and delay the onset of SREs in LADC patients regardless of mutational status (7). Bisphosphonates are specific inhibitors of the osteoclast activity thus leading to bone resorption inhibition (8). Moreover, they also decrease osteoblast proliferation and stimulate boneforming and differentiation (8). Furthermore, based on the results of preclinical studies on non-small cell lung cancer (NSCLC) cell lines, bisphosphonate therapy (BTx) seems to have direct antitumor effects as well by inhibiting proliferation, inducing apoptosis, and modulating the immune microenvironment (9). Besides BTx, radiation therapy (RTx) is also frequently used in bone metastatic LADC for stabilization of impending pathologic fractures and treatment or prevention of spinal cord compression, pathologic fractures and bone pain (10).

In the era of precision medicine, oncogenic driver mutations have a major impact on the therapeutic strategies in LADC (11). Importantly, the most common gain-offunction alterations in LADC are the carcinogenic Kirsten rat sarcoma viral oncogene homolog (KRAS) mutations, accounting for approximately $25 \%$ to $30 \%$ of all LADCs in Western countries and about $10-15 \%$ in Asian patients
$(12,13)$. Although the role of KRAS mutations in LADC is intensely investigated, its prognostic and predictive power in these patients remains controversial (11). Initially, KRAS mutations have been defined as a negative prognostic factor with unfavorable survival rates and disease-free survival compared to KRAS wild-type (WT) tumors (14-16). These results were further supported by the results of two metaanalyses also concluding that KRAS mutation is a negative prognosticator in $\operatorname{LADC}(17,18)$. Meanwhile, a more recent comprehensive study including more than 1,500 NSCLC patients from four trials of adjuvant chemotherapy (CTx) concluded that KRAS mutation has no clear prognostic or predictive relevance (19). Altogether, its general prognostic role is rather controversial, and emerging evidence suggests that the metastatic site-specific variations in LADC might as well influence the prognostic importance and potential clinical relevance of KRAS mutation (20). Accordingly, our group previously found that the presence of KRAS mutations in bone metastatic LADC patients might indeed be associated with significantly worse outcomes, but limited data is available regarding the association between KRAS mutational status and the impact of therapeutic approaches in these patients (20). Therefore, in order to better understand the influence of KRAS mutational status on therapeutic approaches, our aim was to investigate the role of BTx and RTx in KRAS WT vs. KRAS mutant LADC patients diagnosed with bone metastasis.

We present the following article in accordance with the REMARK reporting checklist (available at http://dx.doi. org/10.21037/tlcr-20-754).

\section{Methods}

\section{Ethics statement}

Our research was conducted in accordance with the guidelines of the Helsinki Declaration (as revised in 2013) of the World Medical Association and with the approval of the national level ethics committee (Hungarian Scientific and Research Ethics Committee of the Medical Research Council, 52614-4/2013/EKU), which waived the need for individual informed consent for this retrospective study.

\section{Study population}

Consecutive patients who were diagnosed with histologically confirmed LADC and simultaneous bone metastasis at the National Institute of Oncology, Budapest, Hungary and 
National Korányi Institute of Pulmonology, Budapest, Hungary between January 1998 and November 2013 were included in this study. Of note, none of the included patients presented any other distant organ metastases at the time of diagnosis. Tumor tissue samples for routine histopathologic examination and molecular pathology testing were obtained via endobronchial biopsy or CT guided lung biopsy. The bone metastases were identified by CT scan, PET-CT or MRI of the skeleton. The demographic and clinicopathological characteristics of the patients including gender, age, CTx, BTx and RTx, KRAS mutational status and OS were retrospectively collected. OS was estimated from the time of diagnosis of bone metastasis, until death, or last available follow-up, performed in July 2013. TNM stage according to the Union for International Cancer Control (7th edition) was also recorded at the time of diagnosis (21).

\section{KRAS mutation analysis}

For the current study, all mutational analyses were performed at the 2nd Department of Pathology of the Semmelweis University or at the National Institute of Oncology, as previously described (22). In brief, tumorrich area on $\mathrm{H} \& \mathrm{E}$ staining was specifically determined by pathologists prior to macrodissection from the formalin fixed paraffin-embedded (FFPE) tissue samples to include predominantly tumor cells without significant necrosis or inflammation. Based on the validated instructions of the manufacturer, DNA was extracted using the MasterPure ${ }^{\mathrm{TM}}$ DNA Purification Kit (Epicentre Biotechnologies, WI). KRAS mutational status was screened by a microfluid-based restriction fragment detection system characterized by $5 \%$ mutant tumor cell content sensitivity $(22,23)$.

\section{Treatment}

According to the National Comprehensive Cancer Network (NCCN) clinical practice guidelines (24), only Eastern Cooperative Oncology Group (ECOG) performance status (PS) 0 or 1 LADC patients were included, since higher PS contraindicates the use of cytotoxic CTx and RTx. Drug administration was performed in accordance with contemporary NCCN guidelines and the Hungarian health care financial regulations. Regarding CTx, patients were treated with platinum-based combination CTx either with paclitaxel and carboplatin (PC), gemcitabine and cisplatin (GC), or etoposide and cisplatin (EC). RTx mainly included palliative external beam RTx. BTx included either first-generation bisphosphonate clodronate, or secondgeneration bisphosphonate pamidronate or zoledronic acid administered intravenously in 4-week cycles.

\section{Statistical analysis}

Patients were grouped according to their KRAS mutational status (WT KRAS and KRAS mutant) and treatment (CTx, RTx or BTx). The correlation of clinicopathological parameters with KRAS status and therapeutic modalities was analyzed by Chi square test. OS was demonstrated by Kaplan-Meier curves and for univariate analysis both Mantel-Cox and Gehan-Breslow-Wilcoxon tests were used. Multivariate analysis was performed using a Cox regression model. Metric data is shown as median or mean and corresponding range or as median and corresponding 95\% CI in case of OS. Differences between groups were considered to be statistically significant at a $\mathrm{P}$ value of $<0.05$. Statistical analyses were performed with GraphPad Prism 5.0 (GraphPad Software, Inc., San Diego, CA, USA) and with the PASW Statistics 24.0 package (Predictive Analytics Software, SPSS Inc., Chicago, IL, USA).

\section{Results}

\section{Patient characteristics and KRAS mutational status}

A total of 134 patients diagnosed with LADC and simultaneous bone metastasis were included in this study as shown in Table 1. Ninety-three patients of the full cohort were identified as KRAS WT (69.4\%) and 41 (30.6\%) as KRAS mutant patients. The mean age of patients with KRAS mutation was found to be significantly lower than those with WT KRAS (58.9 vs. 62.9, respectively; $\mathrm{P}=0.029$; Figure $1 A)$. Eighty-three patients $(62 \%)$ received BTx and the mean age was significantly lower among patients with BTx than among those without BTx (mean age $60.3 \pm 9.2 v s$. $64.0 \pm 10.3$, respectively; $\mathrm{P}=0.03$; Figure $1 B$ ). With regards to specific bisphosphonate agents 37,9 and 28 patients received clodronate, pamidronate and zoledronic acid, respectively. Of note, no data was available on the exact type of administered bisphosphonate agent in 9 cases. Our cohort consisted of 85 male and 49 female patients and no significant association was observed between gender and mutational status or therapeutic modality. KRAS mutation showed no association with ECOG score. The administration of RTx or BTx was also not significantly associated with KRAS 
Table 1 Patient characteristics grouped by KRAS mutation and bisphosphonate treatment

\begin{tabular}{|c|c|c|c|c|c|c|c|}
\hline Characteristics & All patients & \multicolumn{3}{|c|}{ KRAS status } & \multicolumn{3}{|c|}{ Bisphopshonate therapy } \\
\hline Total & $134(100 \%)$ & $93(69.4 \%)$ & $41(30.6 \%)$ & & $83(61.9 \%)$ & $51(38.1 \%)$ & \\
\hline Age (mean $\pm \mathrm{SD}$ ) & $61.7 \pm 9.8$ & $62.9 \pm 9.4$ & $58.9 \pm 10.2$ & 0.029 & $60.3 \pm 9.2$ & $64.0 \pm 10.3$ & 0.03 \\
\hline Gender & & & & 0.25 & & & 0.86 \\
\hline Male & $85(63.5 \%)$ & $62(66.7 \%)$ & $23(56.1 \%)$ & & $52(62.7 \%)$ & $33(64.7 \%)$ & \\
\hline ECOG & & & & 0.7 & & & $<0.0001$ \\
\hline 0 & $84(62.7 \%)$ & 57 (61.3\%) & $27(65.8 \%)$ & & $64(77.1 \%)$ & $20(39.2 \%)$ & \\
\hline 1 & $50(37.3 \%)$ & $36(38.7 \%)$ & $14(34.2 \%)$ & & 19 (22.9\%) & $31(60.7 \%)$ & \\
\hline No & $81(60.5 \%)$ & $59(63.5 \%)$ & $22(53.7 \%)$ & & $43(51.8 \%)$ & $38(74.5 \%)$ & \\
\hline
\end{tabular}

KRAS, Kirsten rat sarcoma viral oncogene homolog; ECOG, Eastern Cooperative Oncology Group; SD, standard deviation.
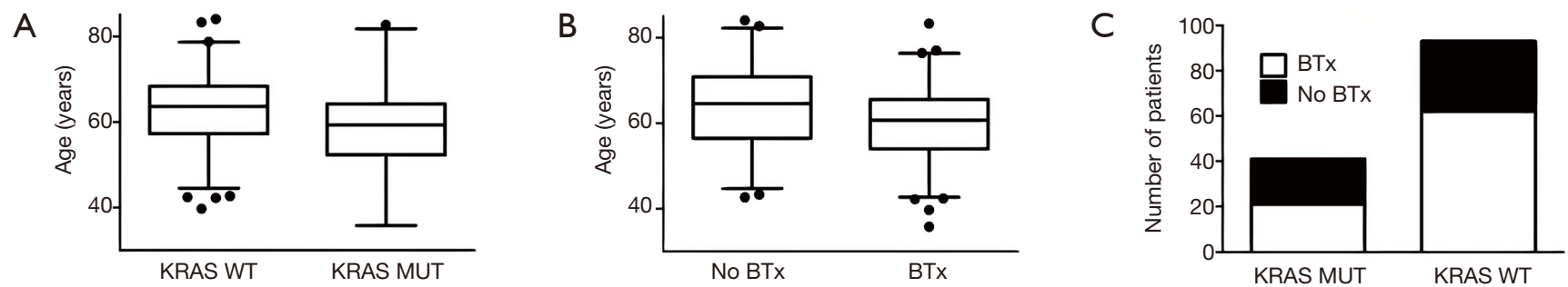

Figure 1 Patient characteristics according to KRAS mutational status and therapeutic modalities. (A) The mean age of patients with KRAS mutation was significantly lower than those with WT KRAS (58.9 vs. 62.9, respectively; $\mathrm{P}=0.029)$. (B) Patients treated with BTx had a

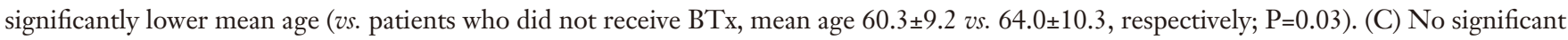
association was observed between KRAS mutational status and the administration of BTx. BTx, bisphosphonate therapy; WT, wild-type.

mutational status (Table 1 and Figure $1 C$, respectively). In contrast, patients receiving BTx were significantly more likely to have ECOG 0 and RTx (Table 1).

\section{KRAS mutation associates with inferior OS}

The median OS for the entire cohort was 7.8 months. Patients with KRAS WT tumors had a significantly longer median OS compared to those with KRAS mutation (10.2 vs. 5.1 months, respectively; Figure 2A, Table 2). With regards to combination CTx, no significant differences in OS have been observed in patients treated with PC $v s$. GC or EC ( $\mathrm{P}=0.297$, Figure S1). In contrast, Kaplan-Meyer curves demonstrated longer median OS in patients who received BTx (10.1 vs. 4.3 months in BTx-naive patients; Figure 2B). Notably, patients receiving second-generation BTx exhibited significantly superior OS compared to those receiving first-generation $\mathrm{BTx}$ (median OSs were $13.2 v s$. 7.1 months, respectively; $\mathrm{P}=0.041$; Figure $\mathrm{S} 2$ ). In regards with RTx, the median OS was higher among the patients receiving RTx compared to RTx-naive patients (11 vs. 5.9 months, Figure 2C). Importantly, the difference in survival between the groups dichotomized by therapeutic modalities disappears for the late events (Figure 2B,C), accordingly only the Gehan-Breslow-Wilcoxon tests indicate significant differences. In contrast, KRAS mutational status curves remain separated for the entire survival range and thus KRAS status has a highly significant impact on survival both by 

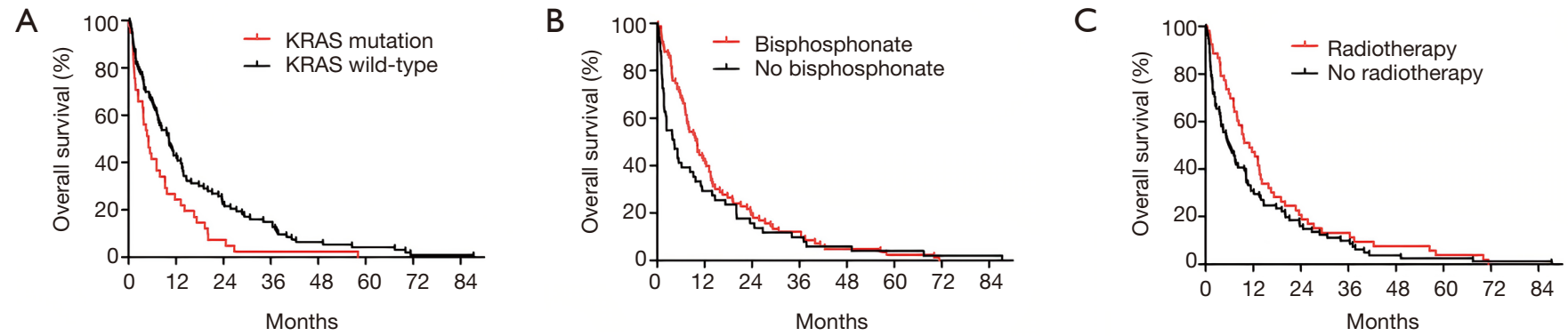

Figure 2 Kaplan-Meier estimates for OS in bone metastatic LADC patients according to KRAS mutational status and therapeutic modalities including BTx and RTx. (A) LADC patients with tumors harboring KRAS mutations had significantly shorter median OS than those with KRAS WT tumors (median OSs were 5.1 vs. 10.2 months, respectively; $\mathrm{P}=0.008$ ). (B) Patients receiving BTx had significantly increased median OS (vs. BTx-naive patients; median OS were 10.1 vs. 4.3 months, respectively, $\mathrm{P}=0.007)$. (C) Similarly, median OS was also significantly increased in LADC patients receiving RTx compared to those who did not receive RTx (median OSs were 11 vs. 5.9 months, respectively $\mathrm{P}=0.021$ ). BTx, bisphosphonate therapy; LADC, lung adenocarcinoma; OS, overall survival; RTx, radiation therapy; WT, wild-type.

Table 2 Prognostic impact of KRAS mutation, radiotherapy and bisphosphonate treatment

\begin{tabular}{|c|c|c|c|c|c|c|c|}
\hline \multirow{2}{*}{ Variable } & \multirow{2}{*}{ OS (months) } & \multicolumn{3}{|c|}{ Univariable analysis } & \multicolumn{3}{|c|}{ Multivariable analysis } \\
\hline & & HR & $95 \% \mathrm{Cl}$ & $P^{*}$ & HR & $95 \% \mathrm{Cl}$ & $P$ \\
\hline KRAS status & & & $0.349-0.820$ & $0.008^{*}$ & & $0.382-0.833$ & 0.004 \\
\hline Wt & 10.2 & 1 & & & 1 & & \\
\hline Mutant & 5.1 & 0.535 & & & 0.564 & & \\
\hline Yes & 11.0 & 1 & & & 1 & & \\
\hline No & 5.9 & 0.763 & & & 0.737 & & \\
\hline Bisphosphonate therapy & & & $0.541-1.127$ & $0.007^{*}$ & & $0.647-1.404$ & 0.810 \\
\hline Yes & 10.1 & 1 & & & 1 & & \\
\hline
\end{tabular}

*, Gehan-Breslow-Wilcoxon test. KRAS, Kirsten rat sarcoma viral oncogene homolog; OS, overall survival; wt, wild-type; HR, hazard ratio;

$\mathrm{Cl}$, confidence interval.

Mantel-Cox and Gehan-Breslow-Wilcoxon tests. Following univariate analysis of the impact of KRAS mutation, RTx and BTx we performed a multivariate analysis using these three factors. The presence of KRAS mutation remained a significant predictor of shorter OS.

\section{KRAS mutation confers inferior outcome in BTx or RTx subgroups}

Next, we investigated whether KRAS mutation remains a significant prognosticator in the subgroups of patients receiving BTx or RTx. We found that the OS was significantly higher in the KRAS WT BTx group (vs. the
KRAS mutant BTx group; the median OSs were 11 vs. 5.8 months, respectively; $\mathrm{P}=0.023$; Figure $3 A$ ). Similarly, KRAS mutation was a strong prognostic factor in the cohort of patients who received RTx (median OS KRAS WT vs. KRAS mutant were 13.5 vs. 7 months, respectively; $\mathrm{P}=0.0168$, Figure 3B).

Importantly, we also found that in the KRAS WT subgroup patients with BTx had significantly increased OS compared to patients without BTx (median OSs were 11 vs. 5.2 months, respectively; $\mathrm{P}=0.032$, Gehan-BreslowWilcoxon test; Figure 3A). As for patients with KRAS mutant tumors, the difference in median OS between patients with or without BTx did not reach statistical 

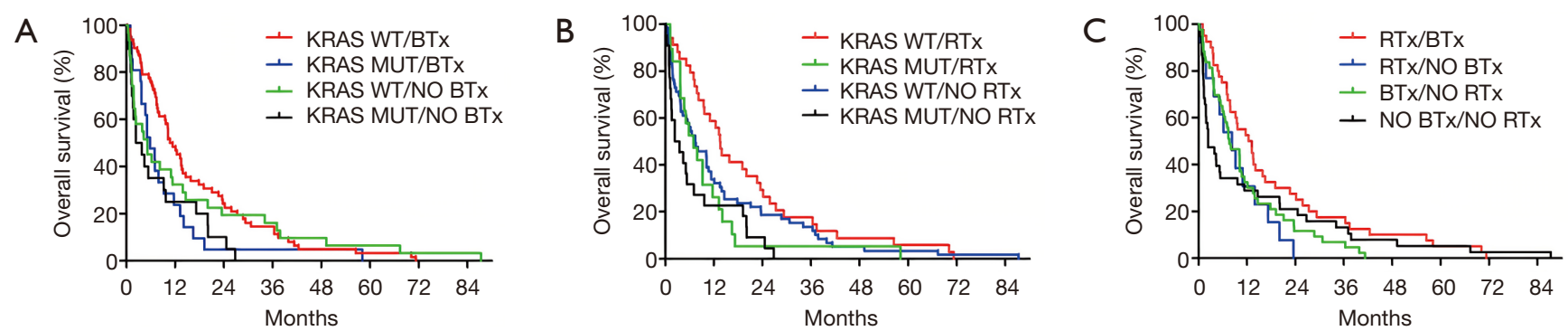

Figure 3 Kaplan-Meier estimates for OS in bone metastatic LADC patients according to KRAS mutational status and specific therapeutic approaches. (A) LADC patients with KRAS WT tumors receiving BTx had significantly increased median OS (vs. those with KRAS mutant tumors treated with BTx, median OSs were 11 vs. 5.8 months, respectively; $\mathrm{P}=0.023)$. With regards to KRAS mutational status, in KRAS WT LADC patients the median OS was significantly increased in patients receiving BTx compared to BTx-naive patients (median OSs were 11 vs. 5.2 months, respectively; $\mathrm{P}=0.032$ ). In contrast, no significant differences in OS have been observed in KRAS-mutant LADC patients with or without BTx (median OSs were 5.8 vs. 3.1 months, respectively; $\mathrm{P}=0.35$ ). (B) RTx-treated patients with KRAS WT tumors exhibited significantly superior OS compared to those with KRAS-mutant tumors (median OSs were 13.5 vs. 7 months, respectively; $\mathrm{P}=0.016$ ). According to KRAS mutational status, in patients with KRAS WT tumors, RTx conferred a significant benefit for OS when compared to patients not receiving RTx (median OS; 13.6 vs. 7.4 months; $\mathrm{P}=0.031$ ). The median OS did not differ significantly in KRAS-mutant LADC patients treated with or without RTx (median OSs were 7 vs. 3 months; $\mathrm{P}=0.12$ ). (C) LADC patients receiving both RTx and BTx had significantly improved OS compared to those who received only RTx or BTx or none of the aforementioned modalities $(\mathrm{P}=0.031)$. BTx, bisphosphonate therapy; LADC, lung adenocarcinoma; OS, overall survival; RTx, radiation therapy; WT, wild-type.

significance (median OSs were 5.8 vs. 3.1 months, respectively; $\mathrm{P}=0.35$; Figure $3 A$ ).

Next, we evaluated the effects of RTx in the KRAS mutational status subgroups. In the KRAS WT subgroup, RTx conferred a significant benefit for OS when compared to patients not receiving RTx (median OS; 13.6 vs. 7.4 months; $\mathrm{P}=0.032$; Figure $3 B$ ). As for the patients with KRAS mutation, the median OS difference was not statistically significant (7 months for RTx and 3 months for patients without RTx; $\mathrm{P}=0.12$; Figure $3 B$ ).

\section{Interaction of radiation therapy and bisphosphonate treatment}

Finally, when evaluating the interaction between BTx and RTx irrespective of KRAS mutational status, we found that patients who received both BTx and RTx had a significantly longer OS compared to those who received only BTx or RTx or none of the aforementioned modalities $(\mathrm{P}=0.031$; Figure 3C).

\section{Discussion}

Despite major improvements and various new treatment modalities for advanced-stage lung cancer, patients with bone metastasis still have a rather poor prognosis $(5,25)$.
The median survival is usually less than 1 year from diagnosis of bone metastasis $(5,26)$. In addition, skeletal metastases are often complicated by metabolic disorders such as hypercalcemia, pathologic fractures, and spinal cord compression $(7,27)$. These skeletal related events (SREs) can substantially reduce the quality of life and increase the economic burden (27).

KRAS is the most frequently mutated oncogene in LADC in Western countries, yet limited data is available regarding the clinical relevance of KRAS mutation in LADC patients diagnosed with bone metastases. Therefore, in this cross-sectional retrospective study of bone metastatic LADC patients we evaluated the effects of KRAS mutational status on OS according to BTx and RTx.

In the current cohort of 134 Caucasian patients, we detected $30.6 \%$ KRAS mutation frequency which is similar to previous findings of Lohinai et al., Confavreux et al., and Bittner et al. $(20,28,29)$. Of note, the KRAS mutation rate in the presented cohort is also similar to the general incidence of KRAS mutation in Caucasian LADC patients (12). Whether the incidence of KRAS mutation is associated with skeletal metastases remains an open question. While Zhao et al. found that patients with KRAS-mutated tumors had a higher incidence of bone metastasis than those with the WT gene, others found no significant association between KRAS mutational status and the appearance of skeletal 
metastases (30-32). Notably, however, the molecular driver subtypes might also play a key role in the appearance of bone metastases, since Kuijpers et al. found that only patients with KRAS G12A mutation had a higher incidence of bone metastases (33). With regards to the effects of KRAS mutational status on survival, we found that patients with KRAS WT tumors had significantly improved median OS than those with KRAS mutant tumors. This observation is in line with data previously reported by us in another large LADC study (20). Nevertheless, it is also important to mention that to date, the prognostic relevance and thus the clinical utility of KRAS oncogenic mutations in LADC in general is still controversial, partly due to vast heterogeneity of the studies in terms of ethnicity, tumor stage, and treatment modality $(11,22,34)$. In our study, however, a relatively homogeneous patient cohort was used, providing evidence that KRAS mutation is indeed a negative prognosticator in advanced-stage LADC patients with bone metastases. In support of this, multivariate analysis also confirmed the role of KRAS mutation as an independent negative prognostic factor in these patients. Importantly, the prognostic relevance of KRAS mutational status was not influenced by the therapeutic approaches, since WT KRAS status was associated with improved OS both in BTx- and RTx-treated patients. To the best of our knowledge, ours is the first detailed evaluation of the prognostic relevance of KRAS mutational status in bone metastatic LADC patients with regards to specific therapeutic approaches including BTx and RTx.

Next, we investigated if KRAS mutational status had an impact on response to BTx and RTx. Bisphosphonates are synthetic analogues of pyrophosphate, a natural regulator of bone metabolism, which inhibits osteoclastmediated bone resorption, decrease osteoblast proliferation and stimulate bone-forming and differentiation $(7,35)$. In addition, BTx also have direct antitumor effects by inhibiting proliferation, inducing apoptosis, and modulating the immune microenvironment in breast cancer, pancreatic cancer, prostate cancer, and NSCLC under both in vitro and in vivo conditions (9,36-39). Meanwhile, RTx mainly consists of external beam radiation and is performed primarily to relieve pain, prevent pathologic fractures and spinal cord compression, and consequently to maintain the patient's quality of life $(40,41)$. Altogether, both BTx and RTx plays a crucial role in the management of bone metastases in LADC $(41,42)$. Notably, in our cohort, KRAS WT LADC patients treated with BTx and RTx indeed had significantly improved OS than BTx-and RTx-naive patients, respectively. In contrast, however, neither BTx, nor RTx conferred a significant benefit for OS in patients with KRAS mutant tumors. As reported by a preclinical study, a possible explanation of this observation might be that BTx including zoledronic acid is unable to inhibit the prenylation of mutant KRAS unlike in the case of WT KRAS (43). Therefore, KRAS WT tumor cells are more likely to be inhibited by BTx, leading to reduced proliferation capacity (43). To our knowledge, this is the first study indicating distinct BTx and RTx efficacy with regards to KRAS mutational status in clinical setting. Therefore, our finding supports the proposal that KRAS mutational status should be taken into account when considering BTx and RTx in bone metastatic LADC patients.

Finally, we investigated the distinct effects of BTx and RTx on survival irrespective of KRAS mutational status and found that both therapeutic modalities improve the median OS. In addition, we also found that patients who received both BTx and RTx had longer OS compared to those who received only BTx or RTx or none of the aforementioned modalities. This finding is in line with the results of preclinical studies on multiple myeloma, breast-, prostate- and small cell lung cancer (44-46). The distinct mechanisms for the interaction of systemic BTx with $\mathrm{RTx}$ were originally described by Hoskin and Steel $(35,47)$. Accordingly, a possible explanation which lies behind the additive and superadditive effect of these two treatment modalities might be that both BTx and RTx have mostly effect upon osteoclast activity (35). Consequently, through their common action on osteoclasts, a positive interaction in the affected area might be suspected (35). Moreover, based on cell line data, BTx such as zoledronic acid and RTx might also cause DNA damage and intensify cytotoxic activity when given together (48). Yet, to date, the exact mechanisms behind the additive and superadditive effect of BTx and RTx are still rather unknown (48).

The present study had certain limitations given by its retrospective nature. First, no information was available on the exact dose and cycles of the administered BTx and RTx. Due to the relatively long time period, diagnostic methods and treatment guidelines may have changed over the years which might also influence the prognosis. Another limitation was the lack of detailed clinicopathological data regarding disease history, other co-morbidities, and tumor characteristics. Of note, data on detailed smoking history, which may be associated with substitution-specific KRAS mutational status, was also not fully available in our cohort. In addition, as significantly more patients with 
ECOG PS score 0 received BTx (vs. ECOG 1 patients), the results of the univariate analysis with regards to the efficacy of BTx might be biased. Importantly, however, ECOG 0 and 1 patients typically have similar survival outcomes (both subgroups being labeled as having "good" PS for clinical research purposes) (49). Molecular methods were focusing on the presence or absence of KRAS mutations and the KRAS WT cohort was not analyzed for additional oncogenic driver mutations. However, all EGFR mutant cases were excluded from the study. Additionally, the final number of included patients was relatively small due to our strict inclusion criteria. Nevertheless, this approach enabled us to analyze a homogenous cohort of at-diagnosis bone metastatic lung cancer patients with the same ethnicity, histology and disease stage. Finally, data was not available on specific KRAS mutational subtypes for each case preventing us from a subtype-specific analysis. Thus, altogether, to address the above limitations, our findings require independent confirmation in cohorts with larger available datasets on clinicopathological data.

\section{Conclusions}

In summary, our results indicate that KRAS mutation is a negative prognosticator in at-diagnosis bone metastatic LADC regardless of BTx and RTx. Furthermore, this is the first study that comprehensively evaluates the effects of BTx and RTx with respect to KRAS mutational status. Accordingly, both BTx and RTx can increase the OS with a pronounced benefit for patients with KRAS WT tumors. Of note, our study also suggests that the concomitant use of BTx and RTx might increase the OS irrespective of KRAS mutational status compared to those receiving only BTx, RTx, or none of the aforementioned therapeutic modalities. Altogether, our findings might not only help to improve the efficacy of BTx and RTx in bone metastatic LADC patients by improving patient selection but might as well contribute to the development of new therapeutic approaches with regards to KRAS mutational status.

\section{Acknowledgments}

The authors thank the patients and the clinical teams involved.

Funding: BD, JM and JT acknowledge funding from the Hungarian National Research, Development and
Innovation Office (NAP2-2017-1.2.1- NKP-0002 and K129065 to BD and JM, KNN121510 and KH130356 to BD and K116151, NVKP16-1-2016-0004 and NVKP16-1-2016-0020 to JT). BD and VL were also supported by the Austrian Science Fund (FWF I3522, FWF I3977 and I4677). VL is a recipient of Janos Bolyai Research Scholarship of the Hungarian Academy of Sciences and the UNKP-19-4 New National Excellence Program of the Ministry for Innovation and Technology. ZM was supported by the UNKP-20-3 New National Excellence Program of the Ministry for Innovation and Technology.

\section{Footnote}

Reporting Checklist: The authors have completed the REMARK reporting checklist. Available at http://dx.doi. org/10.21037/tlcr-20-754

Peer Review File: Available at http://dx.doi.org/10.21037/ tlcr-20-754

Conflicts of Interest: All authors have completed the ICMJE uniform disclosure form (available at http://dx.doi. org/10.21037/tlcr-20-754). The authors have no conflicts of interest to declare.

Ethical Statement: The authors are accountable for all aspects of the work in ensuring that questions related to the accuracy or integrity of any part of the work are appropriately investigated and resolved. Our research was conducted in accordance with the guidelines of the Helsinki Declaration (as revised in 2013) of the World Medical Association and with the approval of the national level ethics committee (Hungarian Scientific and Research Ethics Committee of the Medical Research Council, 52614-4/2013/EKU), which waived the need for individual informed consent for this retrospective study.

Open Access Statement: This is an Open Access article distributed in accordance with the Creative Commons Attribution-NonCommercial-NoDerivs 4.0 International License (CC BY-NC-ND 4.0), which permits the noncommercial replication and distribution of the article with the strict proviso that no changes or edits are made and the original work is properly cited (including links to both the formal publication through the relevant DOI and the license). See: https://creativecommons.org/licenses/by-nc-nd/4.0/. 


\section{References}

1. Bray F, Ferlay J, Soerjomataram I, et al. Global cancer statistics 2018: GLOBOCAN estimates of incidence and mortality worldwide for 36 cancers in 185 countries. CA Cancer J Clin 2018;68:394-424.

2. Zappa C, Mousa SA. Non-small cell lung cancer: current treatment and future advances. Transl Lung Cancer Res 2016;5:288-300.

3. Rinaldi S, Berardi R. Lung cancer prognosis: can histological patterns and morphological features have a role in the management of lung cancer patients? Ann Transl Med 2017;5:353.

4. Jones GS, Baldwin DR. Recent advances in the management of lung cancer. Clin Med (Lond) 2018;18:s41-6.

5. Zhou Y, Yu QF, Peng AF, et al. The risk factors of bone metastases in patients with lung cancer. Sci Rep 2017;7:8970.

6. Klikovits T, Lohinai Z, Fabian K, et al. New insights into the impact of primary lung adenocarcinoma location on metastatic sites and sequence: A multicenter cohort study. Lung Cancer 2018;126:139-48.

7. D'Antonio C, Passaro A, Gori B, et al. Bone and brain metastasis in lung cancer: recent advances in therapeutic strategies. Ther Adv Med Oncol 2014;6:101-14.

8. Al Husaini H, Wheatley-Price P, Clemons M, et al. Prevention and Management of Bone Metastases in Lung Cancer: A Review. J Thorac Oncol 2009;4:251-9.

9. Green JR. Antitumor effects of bisphosphonates. Cancer 2003;97:840-7.

10. Fairchild A. Palliative radiotherapy for bone metastases from lung cancer: Evidence-based medicine? World J Clin Oncol 2014;5:845-57.

11. Tímár J. The clinical relevance of KRAS gene mutation in non-small-cell lung cancer. Curr Opin Oncol 2014;26:138-44.

12. Ferrer I, Zugazagoitia J, Herbertz S, et al. KRAS-Mutant non-small cell lung cancer: From biology to therapy. Lung Cancer 2018;124:53-64.

13. Yang H, Liang SQ, Schmid RA, et al. New Horizons in KRAS-Mutant Lung Cancer: Dawn After Darkness. Front Oncol 2019;9:953.

14. Kern JA, Slebos RJ, Top B, et al. C-erbB-2 expression and codon $12 \mathrm{~K}$-ras mutations both predict shortened survival for patients with pulmonary adenocarcinomas. J Clin Invest 1994;93:516-20.

15. Mitsudomi T, Steinberg SM, Oie HK, et al. ras gene mutations in non-small cell lung cancers are associated with shortened survival irrespective of treatment intent. Cancer Res 1991;51:4999-5002.

16. Slebos RJ, Kibbelaar RE, Dalesio O, et al. K-ras oncogene activation as a prognostic marker in adenocarcinoma of the lung. N Engl J Med 1990;323:561-5.

17. Huncharek M, Muscat J, Geschwind JF. K-ras oncogene mutation as a prognostic marker in non-small cell lung cancer: a combined analysis of 881 cases. Carcinogenesis 1999;20:1507-10.

18. Mascaux C, Iannino N, Martin B, et al. The role of RAS oncogene in survival of patients with lung cancer: a systematic review of the literature with meta-analysis. Br J Cancer 2005;92:131-9.

19. Shepherd FA, Domerg C, Hainaut P, et al. Pooled analysis of the prognostic and predictive effects of KRAS mutation status and KRAS mutation subtype in early-stage resected non-small-cell lung cancer in four trials of adjuvant chemotherapy. J Clin Oncol 2013;31:2173-81.

20. Lohinai Z, Klikovits T, Moldvay J, et al. KRAS-mutation incidence and prognostic value are metastatic site-specific in lung adenocarcinoma: poor prognosis in patients with KRAS mutation and bone metastasis. Sci Rep 2017;7:39721.

21. Mirsadraee S, Oswal D, Alizadeh Y, et al. The 7th lung cancer TNM classification and staging system: Review of the changes and implications. World J Radiol 2012;4:128-34.

22. Cserepes M, Ostoros G, Lohinai Z, et al. Subtype-specific KRAS mutations in advanced lung adenocarcinoma: a retrospective study of patients treated with platinum-based chemotherapy. Eur J Cancer 2014;50:1819-28.

23. Szabó B, Nelhubel GA, Karpati A, et al. Clinical significance of genetic alterations and expression of epidermal growth factor receptor (EGFR) in head and neck squamous cell carcinomas. Oral Oncol 2011;47:487-96.

24. Ettinger DS, Wood DE, Aggarwal C, et al. NCCN Guidelines Insights: Non-Small Cell Lung Cancer, Version 1.2020. J Natl Compr Canc Netw 2019;17:1464-72.

25. Kang EJ, Lee SY, Kim HJ, et al. Prognostic Factors and Skeletal-Related Events in Patients with Small Cell Lung Cancer with Bone Metastases at the Time of Diagnosis. Oncology 2016;90:103-11.

26. Niu YJ, Wen YT, Shen WW, et al. Risk factors for bone metastasis in patients with primary lung cancer: study protocol for a systematic review. BMJ Open 
2014;4:e005202.

27. Cho YJ, Cho YM, Kim SH, et al. Clinical analysis of patients with skeletal metastasis of lung cancer. BMC Cancer 2019;19:303.

28. Confavreux CB, Girard N, Pialat JB, et al. Mutational profiling of bone metastases from lung adenocarcinoma: results of a prospective study (POUMOS-TEC). Bonekey Rep 2014;3:580.

29. Bittner N, Baliko Z, Sarosi V, et al. Bone Metastases and the EGFR and KRAS Mutation Status in Lung Adenocarcinoma--The Results of Three Year Retrospective Analysis. Pathol Oncol Res 2015;21:1217-21.

30. Zhao J, Han Y, Li J, Chai R, et al. Prognostic value of KRAS/TP53/PIK3CA in non-small cell lung cancer. Oncol Lett 2019;17:3233-40.

31. Dormieux A, Mezquita L, Cournede PH, et al. Association of metastatic pattern and molecular status in stage IV non-small cell lung cancer adenocarcinoma. Eur Radiol 2020;30:5021-8.

32. Bittner N, Baliko Z, Sarosi V, et al. The EGFR and KRAS Mutation Status and Correlations with the Prevalence of Bone Metastases - The Results of Three Year Retrospective Analysis. J Carcinog Mutagen 2014;5:5.

33. Kuijpers CC, Hendriks LEL, Derks JL, et al. Association of molecular status and metastatic organs at diagnosis in patients with stage IV non-squamous non-small cell lung cancer. Lung Cancer 2018;121:76-81.

34. Roberts PJ, Stinchcombe TE. KRAS mutation: should we test for it, and does it matter? J Clin Oncol 2013;31:1112-21.

35. Hoskin PJ. Bisphosphonates and radiation therapy for palliation of metastatic bone disease. Cancer Treat Rev 2003;29:321-7.

36. Senaratne SG, Pirianov G, Mansi JL, et al. Bisphosphonates induce apoptosis in human breast cancer cell lines. Br J Cancer 2000;82:1459-68.

37. Tassone P, Tagliaferri P, Viscomi C, et al. Zoledronic acid induces antiproliferative and apoptotic effects in human pancreatic cancer cells in vitro. Br J Cancer 2003;88:1971-8.

38. Lee MV, Fong EM, Singer FR, et al. Bisphosphonate treatment inhibits the growth of prostate cancer cells. Cancer Res 2001;61:2602-8.

39. Di Salvatore M, Orlandi A, Bagalà C, et al. Anti-tumour and anti-angiogenetic effects of zoledronic acid on human non-small-cell lung cancer cell line. Cell Prolif 2011;44:139-46.

40. Kubota H, Soejima T, Sulaiman NS, et al. Predicting the survival of patients with bone metastases treated with radiation therapy: a validation study of the Katagiri scoring system. Radiat Oncol 2019;14:13.

41. De Felice F, Piccioli A, Musio D, et al. The role of radiation therapy in bone metastases management. Oncotarget 2017;8:25691-9.

42. Lopez-Olivo MA, Shah NA, Pratt G, et al. Bisphosphonates in the treatment of patients with lung cancer and metastatic bone disease: a systematic review and meta-analysis. Support Care Cancer 2012;20:2985-98.

43. Kenessey I, Kói K, Horváth O, et al. KRAS-mutation status dependent effect of zoledronic acid in human non-small cell cancer preclinical models. Oncotarget 2016;7:79503-14.

44. Ural AU, Avcu F, Candir M, et al. In vitro synergistic cytoreductive effects of zoledronic acid and radiation on breast cancer cells. Breast Cancer Res 2006;8:R52.

45. Algur E, Macklis RM, Hafeli UO. Synergistic cytotoxic effects of zoledronic acid and radiation in human prostate cancer and myeloma cell lines. Int J Radiat Oncol Biol Phys 2005;61:535-42.

46. Ural AU, Avcu F. Radiosensitizing effect of zoledronic acid in small cell lung cancer. Lung Cancer 2005;50:271-2.

47. Steel GG. The search for therapeutic gain in the combination of radiotherapy and chemotherapy. Radiother Oncol 1988;11:31-53.

48. Ryu K, Murata H, Koto K, et al. Combined effects of bisphosphonate and radiation on osteosarcoma cells. Anticancer Res 2010;30:2713-20.

49. Lilenbaum RC, Cashy J, Hensing TA, et al. Prevalence of poor performance status in lung cancer patients: implications for research. J Thorac Oncol 2008;3:125-9.

Cite this article as: Radeczky P, Megyesfalvi Z, Laszlo V, Fillinger J, Moldvay J, Raso E, Schlegl E, Barbai T, Timar J, Renyi-Vamos F, Dome B, Hegedus B. The effects of bisphosphonate and radiation therapy in bone-metastatic lung adenocarcinoma: the impact of KRAS mutation. Transl Lung Cancer Res 2021;10(2):675-684. doi: 10.21037/tlcr-20-754 


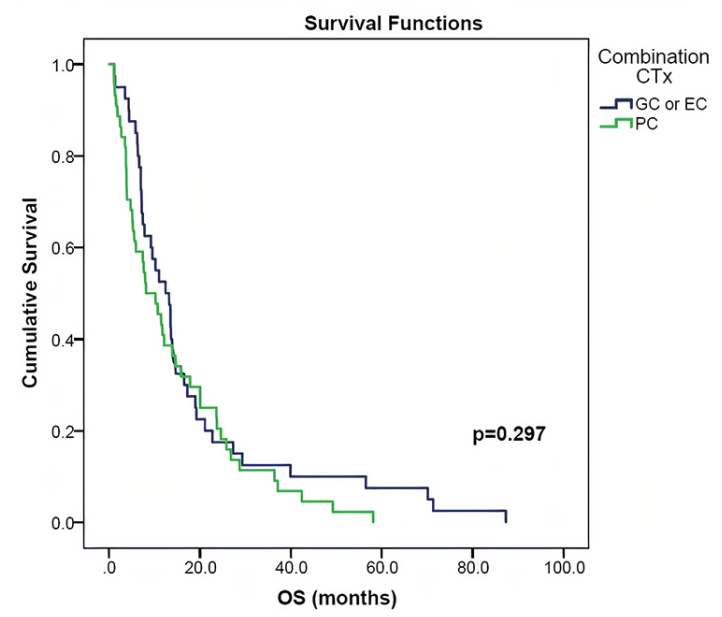

Figure S1 Kaplan-Meier plots for OS in patients with bone metastatic LADC according to combination CTx. The OS did not differ significantly between patients treated with PC $v s$. GC or EC (median OSs were 8.1 vs. 12.4 months, respectively; $\mathrm{P}=0.297$, log-rank test). BTx, bisphosphonate therapy; LADC, lung adenocarcinoma; OS, overall survival; PC, paclitaxel and carboplatin; GC, gemcitabine and cisplatin; EC, etoposide and cisplatin.

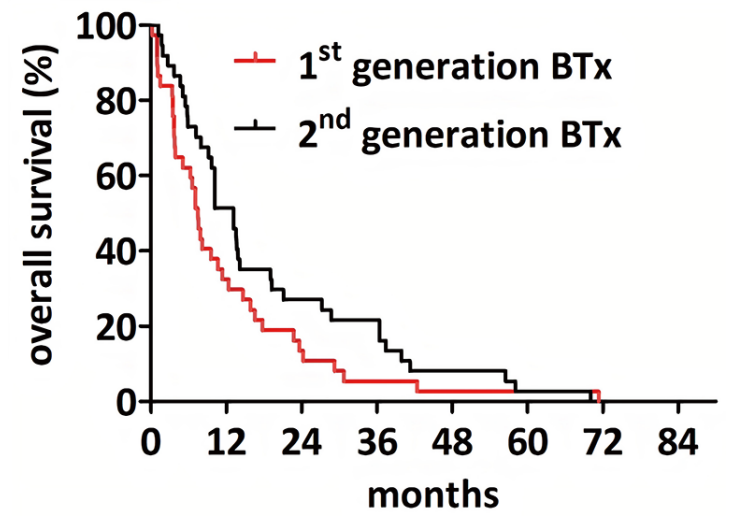

Figure S2 Kaplan-Meier estimates for OS in bone metastatic LADC patients according to different generations of BTx. LADC patients receiving second-generation BTx had significantly increased median OS (vs. those treated with first-generation BTx, median OSs were 13.2 vs. 7.1 months, respectively; $\mathrm{P}=0.041$, Gehan-Breslow-Wilcoxon test). BTx, bisphosphonate therapy; LADC, lung adenocarcinoma; OS, overall survival. 